International Journal of Applied Mathematics

Volume 33 No. $4 \quad 2020,709-731$

ISSN: $1311-1728$ (printed version); ISSN: 1314-8060 (on-line version)

doi: http://dx.doi.org/10.12732/ijam.v33i4.14

\title{
A REINTERPRETATION OF PRINCIPAL COMPONENT ANALYSIS CONNECTED WITH LINEAR MANIFOLDS IDENTIFYING RISKY ASSETS OF A PORTFOLIO
}

\author{
Pierpaolo Angelini \\ Department of Statistical Sciences \\ Sapienza University of Rome \\ 5 Aldo Moro Square, Rome - 00185, ITALY
}

\begin{abstract}
We use the mean-variance model to study a portfolio problem characterized by an investment in two different types of asset. We consider $m$ logically independent risky assets and a risk-free asset. We analyze $m$ risky assets coinciding with $m$ distributions of probability inside of a linear space. They generate a distribution of probability of a multivariate risky asset of order $m$. We show that an $m$-dimensional linear manifold is generated by $m$ basic risky assets. They identify $m$ finite partitions, where each of them is characterized by $n$ incompatible and exhaustive elementary events. We suppose that it turns out to be $n>m$ without loss of generality. Given $m$ risky assets, we prove that all risky assets contained in an $m$-dimensional linear manifold are related. We prove that two any risky assets of them are conversely $\alpha$-orthogonal, so their covariance is equal to 0 . We reinterpret principal component analysis by showing that the principal components are basic risky assets of an $m$-dimensional linear manifold. We consider a Bayesian adjustment of differences between prior distributions to posterior distributions existing with respect to a probabilistic and economic hypothesis.
\end{abstract}

AMS Subject Classification: 51F99, 60B05, 91B06, 91B30, $91 \mathrm{~B} 82$

Key Words: linear manifold; $\alpha$-metric tensor; $\alpha$-orthogonal projection; principal component analysis; proportionality; deviation

Received: May 9, 2020

(c) 2020 Academic Publications 


\section{Introduction}

A risky asset is a random quantity for an investor because he does not know the true value of it. The true value of a random quantity is unique. If an investor calls it random then it is unknown for him. He is therefore in doubt between at least two possible values $([18])$. We suppose that a risky asset is characterized by $n$ possible and distinct monetary values. We consider a finite partition of $n$ incompatible and exhaustive events connected with a risky asset ([7]). They are elementary events. They are points in the space of random quantities, where it is a linear space provided with a quadratic metric. We think of probability as being a mass. It can freely be distributed without altering its geometric support and the measure that appears more natural in the context represented by a linear space. We observe that different distributions of mass are different measures but the notion of measure has no a special status unlike what happens when we refer to measure theory. When we speak about mass as a measure we mean that it can be moved in whatever way we like. When we speak about mass as a measure we do not mean something fixed. Having said that, we consider $m$ risky assets. We suppose that it always turns out to be $n>m$ without loss of generality. We say that $m$ risky assets are logically independent, so there are no circumstances in which the knowledge of some of them can modify the uncertainty concerning the others. This means that there are $n^{m}$ possible values for a multivariate risky asset of order $m$. They coincide with the Cartesian product of the sets of possible values for every risky asset which is the component of a multivariate risky asset of order $m$. Let $X_{i}$ be a generic risky asset. We write $I_{m}=\{1, \ldots, m\}$, so it turns out to be $i \in I_{m}$. The generic and possible value of $X_{i}$ is denoted by $X_{\beta_{i}}$, where we have $\beta_{i} \in I_{n}=\{1, \ldots, n\}$. It follows that if $E_{\beta_{1} \ldots \beta_{m}}$ is a generic event of a finite partition of $n^{m}$ elementary events then we consider an ordered $m$-tuple of corresponding values denoted by $\left(X_{\beta_{1}}, X_{\beta_{2}}, \ldots, X_{\beta_{m}}\right)$. A multivariate risky asset of order $m$ is therefore denoted by

$$
X_{\{m\}}=\left\{\left(X_{\beta_{1}}, X_{\beta_{2}}, \ldots, X_{\beta_{m}}\right) ; p_{\beta_{1} \ldots \beta_{m}} \mid\left(\beta_{1}, \beta_{2}, \ldots, \beta_{m}\right) \in I_{n}^{(m)}\right\},
$$

where we have coherently

$$
\sum_{\beta_{1} \ldots \beta_{m}=1}^{n} p_{\beta_{1} \ldots \beta_{m}}=1 .
$$

In particular, if we deal with a bivariate risky asset then we write

$$
\sum_{\beta_{r} \beta_{s}=1}^{n} p_{\beta_{r} \beta_{s}}=1, \forall(r, s) \in I_{m}^{(2)} .
$$


If the generic and possible value of a bivariate risky asset is denoted by ${ }_{r} X_{\beta}$ then it is also possible to write

$$
\sum_{\beta \gamma=1}^{n}{ }_{r s} p_{\beta \gamma}=1, \forall(r, s) \in I_{m}^{(2)}
$$

instead of (3). We will study mathematical aspects on which a portfolio choice of a risk-averse investor is based ([16]). We will consider a portfolio whose structure is given by $m$ risky assets considered together with a free-risk asset.

\section{Risky assets viewed as elements of a linear manifold}

Let $E^{n}$ be a linear space over $\mathbb{R}$ and let ${ }_{n} B_{\mathbf{e}}^{\perp}=\left\{\mathbf{e}_{\beta} ; \beta \in I_{n}\right\}$ be an orthonormal basis of it. We say that $E^{n}$ is provided with a Euclidean metric because we are able to consider a metric tensor with respect to ${ }_{n} B_{\mathbf{e}}^{\perp}$. It belongs to $E^{n} \otimes E^{n}$. We therefore write

$$
{ }_{\mathbf{e}} g_{\beta \gamma}=\left\langle\mathbf{e}_{\beta}, \mathbf{e}_{\gamma}\right\rangle=\delta_{\beta \gamma},
$$

where $\delta_{\beta \gamma}$ is the Kronecker delta. We have written the generic component of a tensor of order 2. All components of it are scalars. They give origin to an $n \times n$ identity matrix expressed by

$$
\left[\begin{array}{cccc}
1 & 0 & \ldots & 0 \\
0 & 1 & \ldots & 0 \\
\vdots & \vdots & \ddots & \vdots \\
0 & 0 & \ldots & 1
\end{array}\right]
$$

The possible and distinct values of a risky asset are the contravariant components of an $n$-dimensional vector of $E^{n}$ with respect to ${ }_{n} B_{\mathbf{e}}^{\perp}$. We write

$$
\mathbf{x}_{i}=x_{i}^{\beta} \mathbf{e}_{\beta} \in E^{n}
$$

by using the Einstein notation. We consider $m$ risky assets, so we write

$$
\mathbf{x}_{i}=x_{i}^{\beta} \mathbf{e}_{\beta}, \forall i \in I_{m} .
$$

Since it turns out to be $n>m$ we are also able to suppose that all vectors of

(8) are linearly independent. Having said that, we observe that

$$
I_{m}^{[\mathbf{x}]}=\left\{\mathbf{x}_{i} ; i \in I_{m}\right\}
$$


represents a basis of an $m$-dimensional linear manifold embedded in $E^{n}$. It is denoted by ${ }_{\mathrm{x}} V^{m}$. All linear combinations of the basis vectors contained in (9) give origin to the elements of ${ }_{\mathbf{x}} V^{m}$. We consider all their translations with respect to an $n$-dimensional vector of $E^{n}$. It coincides with the zero vector of $E^{n}$. We therefore write

$$
\mathbf{x}=x^{i} \mathbf{x}_{i} \in{ }_{\mathbf{x}} V^{m}, \forall x^{i} \in \mathbb{R}
$$

where we have $i=1, \ldots, m$. We define a risky asset denoted by $X_{i}=\left\{x_{i}^{1}, x_{i}^{2}, \ldots\right.$, $\left.x_{i}^{n}\right\}, i=1, \ldots, m$ : we observe that $x_{i}^{1}$ is the return on $X_{i}$ if $E_{i 1}$ occurs with probability $p_{i 1}, x_{i}^{2}$ is the return on $X_{i}$ if $E_{i 2}$ occurs with probability $p_{i 2}, \ldots, x_{i}^{n}$ is the return on $X_{i}$ if $E_{i n}$ occurs with probability $p_{i n}$. We note that $x_{i}^{1}$ is the wealth that $X_{i}$ yields if $E_{i 1}$ occurs with probability $p_{i 1}, x_{i}^{2}$ is the wealth that $X_{i}$ yields if $E_{i 2}$ occurs with probability $p_{i 2}, \ldots, x_{i}^{n}$ is the wealth that $X_{i}$ yields if $E_{i n}$ occurs with probability $p_{i n}$. It follows that $\overline{\mathbf{x}}_{i}, i=1, \ldots, m$, represents a vector of $E^{n}$ having all its contravariant components equal to the expected return on $X_{i}, i=1, \ldots, m$. It is denoted by $\mathbf{P}\left(X_{i}\right)$, so we write

$$
\overline{\mathbf{x}}_{i}=\left(\begin{array}{c}
\bar{x}_{i}^{1}=\mathbf{P}\left(X_{i}\right) \\
\bar{x}_{i}^{2}=\mathbf{P}\left(X_{i}\right) \\
\vdots \\
\bar{x}_{i}^{n}=\mathbf{P}\left(X_{i}\right)
\end{array}\right)
$$

where we have $i=1, \ldots, m$. We write

$$
\overline{\mathbf{x}}_{i}=\bar{x}_{i}^{\beta} \mathbf{e}_{\beta}, \forall i \in I_{m} .
$$

We observe that every $\overline{\mathbf{x}}_{i}, i=1, \ldots, m$, represents an $n$-dimensional vector of $E^{n}$ with respect to which it is possible to consider all translations of all linear combinations of the basis vectors of ${ }_{\mathrm{x}} V^{m}$ contained in (9). This means that we deal with $m$ linear manifolds, where each of them is denoted by ${ }_{\mathbf{x}_{i}} V^{m}$. It is then possible to obtain the $m$-dimensional linear manifold denoted by ${ }_{\mathbf{d}} V_{(0)}^{m}$. It is expressed by

$$
{ }_{\mathbf{d}} V_{(0)}^{m}={ }_{\mathbf{x}} V^{m} \ominus{ }_{\mathbf{x}} V^{m} .
$$

It is nothing but the direct difference of all linear manifolds denoted by ${ }_{\bar{x}_{1}} V^{m}$, $\ldots, \overline{\mathbf{x}}_{m} V^{m}$. We deal with $m$ summands, where each of them is a difference. The subtrahend of the first difference is always $\overline{\mathbf{x}}_{1}, \ldots$, the subtrahend of the $m$-th difference is always $\overline{\mathbf{x}}_{m}$. We note that the minuends of the first difference coincide with all linear combinations of $\mathbf{x}_{1} \in I_{m}^{[\mathbf{x}]}, \ldots$, the minuends of the $m$ th difference coincide with all linear combinations of $\mathbf{x}_{m} \in I_{m}^{[\mathbf{x}]}$. In particular, 
every vector denoted by $\mathbf{d}_{i} \in{ }_{\mathbf{d}} V_{(0)}^{m}$ represents all deviations of the possible and distinct values of $X_{i}$ from its expected return denoted by $\overline{\mathbf{x}}_{i}, i=1, \ldots, m([24])$. Every vector denoted by $\mathbf{d}_{i} \in{ }_{\mathbf{d}} V_{(0)}^{m}$ therefore represents a basic risky asset. We consequently write

$$
{ }_{(0)} I_{m}^{[\mathbf{d}]}=\left\{\mathbf{x}_{i}-\overline{\mathbf{x}}_{i} ; i \in I_{m}\right\}=\left\{\mathbf{d}_{i} ; i \in I_{m}\right\} .
$$

We observe that (14) represents a basis of ${ }_{\mathrm{d}} V_{(0)}^{m}$. It is also possible to denote it by ${ }_{m} B_{\mathbf{d}}$. All linear combinations of the basic risky assets contained in (14) generate ${ }_{\mathrm{d}} V_{(0)}^{m}$. We consider all their translations with respect to the zero vector of $E^{n}$, so we write

$$
\mathbf{d}=d^{i} \mathbf{d}_{i} \in{ }_{\mathbf{d}} V_{(0)}^{m}, \forall d^{i} \in \mathbb{R},
$$

where we have $i=1, \ldots, m$. We lastly denote by $D_{\{m\}}$ a multivariate risky asset of order $m$ whose components are $m$ risky assets. The first component of $D_{\{m\}}$ is a risky asset whose possible and distinct values coincide with all deviations of $X_{1}$ from $\overline{\mathbf{x}}_{1}, \ldots$, the $m$-th component of $D_{\{m\}}$ is a risky asset whose possible and distinct values coincide with all deviations of $X_{m}$ from $\overline{\mathbf{x}}_{m}$ ([12]). We consequently say that $D_{\{m\}}$ is defined with respect to $X_{\{m\}}$.

\section{A multivariate risky asset and its association probabilities}

Let $\mathbf{y}_{h}$ be a vector representing a risky asset of $D_{\{m\}}$ whose possible and distinct values are obtained by means of a change of origin of the starting values ([11]). We write

$$
\mathbf{y}_{h}=y_{h}^{i} \mathbf{d}_{i} \in{ }_{\mathbf{d}} V_{(0)}^{m} .
$$

We say that $\left\{y_{h}^{i}\right\}$ is a set of $m$ contravariant components of $\mathbf{y}_{h}$ with respect to ${ }_{m} B_{\mathbf{d}}$. Only one contravariant component of $\mathbf{y}_{h}$ is equal to 1 . All other contravariant components of $\mathbf{y}_{h}$ are equal to 0 . Since ${ }_{\mathbf{d}} V_{(0)}^{m}$ is embedded in $E^{n}$ it is possible to write the same vector with respect to ${ }_{n} B_{\mathbf{e}}^{\perp}$. We obtain

$$
\mathbf{y}_{h}=y_{h}^{i} x_{i}^{\beta} \mathbf{e}_{\beta} \in E^{n},
$$

where $\left\{y_{h}^{i} x_{i}^{\beta}\right\}$ is a set of $n$ contravariant components of $\mathbf{y}_{h}$ with respect to ${ }_{n} B_{\mathbf{e}}^{\perp}$. It is evident that $\left\{x_{i}^{\beta}\right\}$ contains all possible and distinct values of a risky asset of $D_{\{m\}}$ with respect to ${ }_{n} B_{\mathbf{e}}^{\perp}$. These values are evidently deviations from a mean value ([8]). It is evident that $\left\{y_{h}^{i}\right\}$ and $\left\{y_{h}^{i} x_{i}^{\beta}\right\}$ are not association probabilities. If we deal with a multivariate risky asset of order $m$ then we have to take association probabilities into account. A multivariate risky asset 
of order $m$ is then characterized by an affine tensor of order $m$ identifying association probabilities. A basis of

$$
\overbrace{E^{n} \otimes E^{n} \otimes \ldots \otimes E^{n}}^{m \text { times }}
$$

is therefore denoted by $B_{n^{m}}=\left\{\mathbf{e}_{\beta_{1}} \otimes \ldots \otimes \mathbf{e}_{\beta_{m}}\right\}$, so we obtain

$$
p_{1 \ldots m}={ }_{1 \ldots m} p^{\beta_{1} \ldots \beta_{m}} \mathbf{e}_{\beta_{1}} \otimes \ldots \otimes \mathbf{e}_{\beta_{m}},
$$

where (18) is the generic component of an affine tensor of order $m$ representing association probabilities whose sum is equal to 1 on the whole partition ([2]). We have to note a very important point: $\mathbf{y}_{h} \in E^{n}$ is nothing but a tensor of order 1 . If we suppose that all contravariant components of an $n$-dimensional vector of $E^{n}$ denoted by $\phi$ are equal to 1 with respect to ${ }_{n} B_{\mathbf{e}}^{\perp}$ then it is possible to construct an affine tensor of order $m-1$ expressed by

$$
\phi_{\beta_{i}}^{\beta_{1} \ldots \beta_{m}}=\prod_{\substack{j=1 \\ j \neq i}}^{m} \phi^{\beta_{j}} .
$$

We construct (19) with respect to $\phi$. Having said that, the following pair of expressions

$$
\left\{\begin{array}{l}
Y_{h}^{\beta_{1} \ldots \beta_{m}}=y_{h}^{i} x_{i}^{\beta_{i}} \phi_{\beta_{i}}^{\beta_{1} \ldots \beta_{m}} \\
{ }_{1 \ldots m} p_{\beta_{1} \ldots \beta_{m}}
\end{array}\right.
$$

allows us of representing all deviations concerning all risky assets of a multivariate risky asset of order $m$ together with their association probabilities. We note that the tensor product between a tensor whose order is equal to 1 and a tensor whose order is equal to $m-1$ is equal to a tensor whose order is equal to $1+m-1=m$. We evidently represent the generic component of an affine tensor of order $m$ identifying association probabilities by means of covariant indices. This is because we will be able to obtain summary statistics about distribution of probability under consideration in this way.

\section{A symmetric tensor obtained by using association probabilities}

If we deal with two risky assets jointly considered then we need to take the tensor product of two linear spaces into account. Let $E^{n} \otimes E^{n}$ be a linear space of affine tensors of order 2 and let $B_{n^{2}}=\left\{\mathbf{e}_{\beta} \otimes \mathbf{e}_{\gamma}\right\}$ be a basis of it. We consider an ordered pair of risky assets denoted by $\left(\mathbf{d}_{i}, \mathbf{d}_{j}\right)$, where every risky 
asset of it belongs to ${ }_{\mathbf{d}} V_{(0)}^{m}$. The affine tensor of order 2 representing association probabilities is expressed by

$$
p_{i j}={ }_{i j} p^{\beta \gamma} \mathbf{e}_{\beta} \otimes \mathbf{e}_{\gamma},
$$

where ${ }_{i j} p^{\beta \gamma}$ is the generic component of it. It is a scalar. It is an association probability. Having said that, we write

$$
{ }_{\mathbf{d} ; \alpha} g_{i j}={ }_{\mathbf{d}} g_{i j}=\left\langle\mathbf{d}_{i}, \mathbf{d}_{j}\right\rangle_{\alpha}=d_{i}^{\beta} d_{j}^{\gamma}{ }_{i j} p_{\beta \gamma}
$$

with respect to ${ }_{m} B_{\mathbf{d}}$. We say that $(22)$ represents a tensor of order 2 called $\alpha$-metric tensor with respect to an $m$-dimensional linear manifold denoted by ${ }_{\mathrm{d}} V_{(0)}^{m}$. It is a symmetric tensor obtained by using association probabilities. Its components are symmetric. If it turns out to be $m>2$ then different comparisons between two risky assets of ${ }_{m} B_{\mathbf{d}}$ are possible. It is essential to note that only pairwise comparisons are possible. This is because we consider a quadratic metric. An $m \times m$ symmetric matrix is then generated by all possible pairwise comparisons. The number of its distinct elements coincides with

$$
C_{m, 2}^{r}=\frac{1}{2} m(m+1),
$$

where every its element is a component of ${ }_{\mathbf{d}} g_{i j}$. In particular, it is possible to derive from (22) the notion of $\alpha$-norm, so we write

$$
{ }_{\mathrm{d}} g_{i i}=\left\|\mathbf{d}_{i}\right\|_{\alpha}^{2}=d_{i}^{\beta} d_{i}^{\beta}{ }_{i} p_{\beta} .
$$

In general, we obtain the following $m \times m$ symmetric matrix

$$
\left[\begin{array}{cccc}
\mathrm{d} g_{11} & \mathbf{d}_{\mathbf{d}} g_{12} & \cdots & { }_{\mathbf{d}} g_{1 m} \\
\mathbf{d} g_{21} & { }_{\mathbf{d}} g_{22} & \cdots & { }_{\mathbf{d}} g_{2 m} \\
\vdots & \vdots & \ddots & \vdots \\
{ }_{\mathbf{d}} g_{m 1} & { }_{\mathbf{d}} g_{m 2} & \cdots & { }_{\mathbf{d}} g_{m m}
\end{array}\right] .
$$

The Schwarz $\alpha$-generalized inequality can be considered in order to complete the $\alpha$-metric structure of ${ }_{\mathrm{d}} V_{(0)}^{m}$. It is given by

$$
\left|\mathbf{d} g_{i j}\right| \leq \sqrt{\mathbf{d} g_{i i}} \sqrt{\mathbf{d} g_{j j}} .
$$

We observe that (5) and (22) are two tensors of order 2. The former is defined with respect to an orthonormal basis of $E^{n}$ without considering association probabilities, while the latter is defined with respect to an ordered pair of basic risky assets of ${ }_{\mathrm{d}} V_{(0)}^{m}$. We note that an affine tensor of order 2 representing 
association probabilities always corresponds to such a pair of risky assets of ${ }_{\mathrm{d}} V_{(0)}^{m}$. Such a tensor must be used in order to obtain (22). It follows that (5) and (22) are conceptually different for this reason. In this context we think of probability as being a non-negative and finitely additive function taking the value 1 on the whole space. On the other hand, there exist situations with respect to which a systematic set-theoretic interpretation of events is not conceptually satisfactory. A single event is then a proposition identified with a real number $([9])$. It is unequivocally stated. It will be true or false at the right time $([4])$.

\section{Eigenvectors connected with a symmetric tensor obtained by using association probabilities and their representation}

Let ${ }_{\mathbf{d}} g_{i j}$ be an $\alpha$-metric tensor connected with ${ }_{\mathbf{d}} V_{(0)}^{m}$ whose components identify (25). We suppose that all entries of (25) outside of its main diagonal are equal to zero. We suppose that all its main diagonal entries are different. We have therefore $m$ different values characterizing the main diagonal entries of (25), where (25) is evidently an $m \times m$ diagonal matrix. We say that ${ }_{\mathrm{d}} g_{i j}$ identifies an eigenequation. The number of its distinct eigenvalues is overall equal to $m$. In other words, given a basis of ${ }_{\mathbf{d}} V_{(0)}^{m}$ consisting of $m$ risky assets of ${ }_{\mathbf{d}} V_{(0)}^{m}$ identifying $m$ marginal distributions of probability, we suppose the following thing: each time we jointly consider two different risky assets of a basis of ${ }_{\mathbf{d}} V_{(0)}^{m}$ we observe that the property of $\alpha$-orthogonality is satisfied ([22]). This means that the covariance of these two risky assets is equal to 0 . We then speak about pairwise non-correlation. Having said that, let ${ }_{m} B_{\mathbf{e}}^{\perp}=\left\{\mathbf{e}_{i} ; i \in I_{m}\right\}$ and ${ }_{m} B_{\mathbf{e}()}^{\perp}=\left\{\mathbf{e}_{(j)} ;(j) \in I_{m}\right\}$ be two distinct orthonormal bases with respect to ${ }_{\mathrm{d}} V_{(0)}^{m}$. We therefore write

$$
\left\langle\mathbf{e}_{i}, \mathbf{e}_{j}\right\rangle=\delta_{i j}
$$

as well as

$$
\left\langle\mathbf{e}_{(i)}, \mathbf{e}_{(j)}\right\rangle=\delta_{(i)(j)} .
$$

If the set of the contravariant components of $\mathbf{e}_{i}$ with respect to ${ }_{m} B_{\mathbf{e}()}^{\perp}$ is denoted by $\left\{A_{i}^{(j)} ;(j) \in I_{m}\right\}$ then we write

$$
\mathbf{e}_{i}=A_{i}^{(j)} \mathbf{e}_{(j)},
$$

where we have $i=1, \ldots, m$. We evidently consider $m$ linear combinations identifying a non-singular matrix denoted by $A=\left\{A_{i}^{(j)} ;(j) \in I_{m}, i \in I_{m}\right\}$. We 
are also able to write

$$
\mathbf{e}_{(k)}=A_{(k)}^{i} \mathbf{e}_{i},
$$

where we have $(k)=1, \ldots, m$. We obtain $A^{-1}=\left\{A_{(k)}^{i} ; i \in I_{m},(k) \in I_{m}\right\}$. This means that it turns out to be

$$
A_{i}^{(j)} A_{(k)}^{i}=\delta_{(k)}^{(j)}
$$

as well as

$$
A_{i}^{(j)} A_{(j)}^{h}=\delta_{i}^{h} .
$$

Let $\mathbf{v}_{(k)}$ be an eigenvector of the $\alpha$-metric tensor whose components identify (25). It is then associated with the eigenvalue denoted by ${ }_{\mathbf{d}} \lambda_{(k)}([21])$. Hence, $\mathbf{v}_{(k)}$ is expressed by

$$
\mathbf{v}_{(k)}=v_{(k)}^{i} \mathbf{e}_{i}
$$

with respect to ${ }_{m} B_{\mathbf{e}}^{\perp}$. Let $\mathbf{v}_{k}$ be an eigenvector of the $\alpha$-metric tensor whose components identify (25). It is then associated with the eigenvalue denoted by ${ }_{\mathbf{d}} \lambda_{k}$. Hence, $\mathbf{v}_{k}$ is expressed by

$$
\mathbf{v}_{k}=v_{k}^{(j)} \mathbf{e}_{(j)}
$$

with respect to ${ }_{m} B_{\mathbf{e}()}^{\perp}$. We observe that $\left\{_{\mathbf{d}} \lambda_{(k)} ;(k) \in I_{m}\right\}$ as well as $\left\{_{\mathbf{d}} \lambda_{k} ; k \in\right.$ $\left.I_{m}\right\}$ are two different enumerations identifying the same eigenvalues. The same eigenvalues are contained in both sets. We are not able to pass from a set to another one because we deal with different enumerations. If $\mathbf{v}_{(k)}$ and $\mathbf{v}_{k}$ are normalized, with $(k)=1, \ldots, m$ as well as $k=1, \ldots, m$, then it turns out to be

$$
\left\langle\mathbf{v}_{(k)}, \mathbf{v}_{(h)}\right\rangle=\delta_{(k)(h)}
$$

and

$$
\left\langle\mathbf{v}_{k}, \mathbf{v}_{h}\right\rangle=\delta_{k h} .
$$

All these eigenvectors are therefore orthonormal. They identify an orthogonal matrix ([14]). We lastly state what it was our intention of showing in this section: all eigenvectors associated with ${ }_{\mathbf{d}} g_{i j}$ are pairwise $\alpha$-orthogonal. Since they are associated with ${ }_{\mathbf{d}} g_{i j}$ we indirectly refer to the property of $\alpha$-orthogonality when we say this thing. 


\section{The projection of a linear manifold onto another one and its reason}

Any evaluation of probability referred to an event always depends on the variable group of circumstances assumed to be relevant to its occurrence ([10]). Such circumstances are known at the time. In general, they vary from moment to moment. This means that any evaluation of probability referred to an event can vary according to the state of knowledge of an investor. It can continuously be enriched by the flow of different information. It can also be enriched by results that are learnt or observed in a gradual way in relation to more or less similar situations and cases ([3]). We therefore consider ${ }_{\mathbf{d}^{\prime}} V_{(0)}^{m}$ for all these reasons. It is an $m$-dimensional linear manifold embedded in $E^{n}$. It is then a translation of an $m$-dimensional linear subspace of $E^{n}$ coinciding with it when one considers a translation with respect to the zero vector of $E^{n}$. It is generated by $m$ vectors, where each of them represents ordered deviations from a mean value subjectively determined by an investor ([5]). They are then linearly independent. They identify $m$ risky assets which are the components of a multivariate risky asset of order $m$ denoted by $D_{\{m\}}^{\prime}$. They identify $m$ marginal probability distributions which are different from the starting ones. We note that all linear combinations of these $m$ basic risky assets generate risky assets which are related. They belong to ${ }_{\mathbf{d}^{\prime}} V_{(0)}^{m}$. This is because they are geometrically represented by $n$-dimensional vectors belonging to the same $m$-dimensional linear subspace of $E^{n}$. If we consider two any risky assets of them together with their association probabilities then we observe that they are $\alpha$-orthogonal, so their covariance is equal to 0 . Given $D_{\{m\}}$, we suppose that every risky asset belonging to $D_{\{m\}}$ is associated with every risky asset belonging to $D_{\{m\}}^{\prime}$. This means that we consider two multivariate risky assets of order $m$ denoted by $D_{\{m\}}$ and $D_{\{m\}}^{\prime}$ as well as a set of $m^{2}$ bivariate risky assets whose generic element is denoted by $\left(D_{i}, D_{j}^{\prime}\right)$. We also consider the $\alpha$ orthogonal projection of ${ }_{\mathbf{d}^{\prime}} V_{(0)}^{m}$ onto ${ }_{\mathbf{d}} V_{(0)}^{m}$. It is denoted by ${ }_{\hat{\mathbf{d}}} V_{(0)}^{m}$. All vectors belonging to ${ }_{\mathrm{d}} V_{(0)}^{m}$ represent the starting risky assets. All vectors belonging to $\hat{\mathrm{d}}_{(0)}^{m}$ express a logical and formal hypothesis with respect to a given structure of the distributions of probability identifying all risky assets under consideration. It is a probabilistic and economic hypothesis. The condition of invariance of the covariance of two risky assets expresses it. Having said that, let $\mathbf{d}_{j}^{\prime}$ be an element of ${ }_{\mathbf{d}^{\prime}} V_{(0)}^{m}$. Let $\hat{\mathbf{d}}_{j}$ be the corresponding element of ${ }_{\hat{\mathbf{d}}} V_{(0)}^{m}$, where we have $j=1, \ldots, m$. We say that it turns out to be

$$
\mathbf{d}_{j}^{*}=\mathbf{d}_{j}^{\prime}-\hat{\mathbf{d}}_{j}, \forall j \in I_{m}
$$


It is therefore possible to construct $\hat{\mathrm{d}}_{(0)}^{m}$ by solving the following system of $m$ linear equations for every value of $j \in I_{m}$. It is given by

$$
\left\langle\mathbf{d}_{j}^{*}, \mathbf{d}_{i}\right\rangle_{\alpha}=0, \forall i \in I_{m}
$$

We consider $m$ systems expressed by (38). It is evident that we are also able to write

$$
\left\langle\mathbf{d}_{j}^{\prime}, \mathbf{d}_{i}\right\rangle_{\alpha}-\left\langle\hat{\mathbf{d}}_{j}, \mathbf{d}_{i}\right\rangle_{\alpha}=0, \forall i \in I_{m} .
$$

We note that (39) tells us that the covariance of two risky assets is invariant. We consider their joint distribution in order to say this thing. We observe that it is possible to write

$$
\hat{\mathbf{d}}_{j}=d_{j}^{h} \mathbf{d}_{h},
$$

where we have $\hat{\mathbf{d}}_{j} \in \hat{\mathbf{d}}_{(0)} V_{(0)}^{m}, j=1, \ldots, m$. We note that it turns out to be $\mathbf{d}_{h} \in{ }_{m} B_{\mathbf{d}}$. We put (40) in (39) as well as we remind how the $\alpha$-metric tensor concerning ${ }_{\mathrm{d}} V_{(0)}^{m}$ has been defined. Hence, we write

$$
\left\langle\mathbf{d}_{j}^{\prime}, \mathbf{d}_{i}\right\rangle_{\alpha}-d_{j \mathbf{d}}^{h} g_{h i}=\left\langle\mathbf{d}_{j}^{\prime}, \mathbf{d}_{i}\right\rangle_{\alpha}-d_{j i}=0 .
$$

It follows that we obtain

$$
d_{j i}=\left\langle\mathbf{d}_{j}^{\prime}, \mathbf{d}_{i}\right\rangle_{\alpha} .
$$

We say that the covariant components of $\hat{\mathbf{d}}_{j}$ with respect to ${ }_{m} B_{\mathbf{d}}$ are expressed by (42). Since the subtrahend of (39) is given by

$$
\left\langle\hat{\mathbf{d}}_{j}, \mathbf{d}_{i}\right\rangle_{\alpha}=d_{j}^{h}\left\langle\mathbf{d}_{h}, \mathbf{d}_{i}\right\rangle_{\alpha}=d_{j \mathbf{d}}^{h} g_{h i}=d_{j i}
$$

we note that the covariant components of $\mathbf{d}_{j}^{\prime}$ and $\hat{\mathbf{d}}_{j}$ with respect to ${ }_{m} B_{\mathbf{d}}$ are obtained in the same way. Having said that, we have to establish the contravariant components of $\hat{\mathbf{d}}_{j}$ in order to complete the $\alpha$-orthogonal projection of ${ }_{\mathbf{d}^{\prime}} V_{(0)}^{m}$ onto ${ }_{\mathbf{d}} V_{(0)}^{m}$. We therefore write

$$
d_{j \mathbf{d}}^{h} g_{h i \mathbf{d}} g^{k i}=d_{j i \mathbf{d}} g^{k i},
$$

so we obtain

$$
d_{j}^{k}=d_{j i} g^{k i}
$$


where the contravariant components of the $\alpha$-metric tensor under consideration denoted by ${ }_{\mathbf{d}} g^{k i}$ are given by

$$
\left[\begin{array}{cccc}
\frac{1}{\mathrm{~d} g_{11}} & 0 & \cdots & 0 \\
0 & \frac{1}{\mathrm{~d} g_{22}} & \cdots & 0 \\
\vdots & \vdots & \ddots & \vdots \\
0 & 0 & \cdots & \frac{1}{\mathrm{~d}^{g_{m m}}}
\end{array}\right] .
$$

We have to note a very important point: we have observed that $m$ linear combinations of the basic risky assets of ${ }_{\mathbf{d}} V_{(0)}^{m}$ give origin to $m$ risky assets of $\hat{\mathbf{d}}_{(0)}^{m}$ represented by $m$ vectors belonging to $\hat{\mathrm{d}}_{(0)}^{m}$ according to (40). Their contravariant components coincide with the coefficients of these $m$ linear combinations. Given such components, it is then possible to obtain the covariant components of the risky assets of $\hat{\mathrm{d}}_{(0)} V^{m}$ by using the covariant components of ${ }_{\mathbf{d}} g_{i j}$ expressed by (25). Given the covariant components of the risky assets belonging to $\hat{\mathrm{d}}_{(0)}^{m}$, it is conversely possible to obtain their contravariant components by using the contravariant components of ${ }_{\mathbf{d}} g^{i j}$ expressed by (46).

\section{A choice of an appropriate basis of a linear manifold: a definition of principal components}

Let $\left\{{ }_{\mathbf{d}} \lambda_{(k)} ;(k) \in I_{m}\right\}$ be the set containing all eigenvalues of the $\alpha$-metric tensor which has been constructed with respect to ${ }_{\mathbf{d}} V_{(0)}^{m}$. We have supposed that they are all distinct. Let $\left\{\mathbf{v}_{(k)} ;(k) \in I_{m}\right\}$ be the corresponding set containing all normalized eigenvectors. They are pairwise $\alpha$-orthogonal because we have supposed that all eigenvalues are distinct. Given $D_{\{m\}}$, we have to note a very important point: we say that the principal components with respect to $D_{\{m\}}$ and denoted by $\mathbf{w}_{(h)},(h)=1, \ldots, m$, are all linear combinations of vectors, where each of them represents a basic risky asset of $D_{\{m\}}$, whose coefficients are the components of a normalized eigenvector ([15]). We therefore write

$$
\mathbf{w}_{(h)}=v_{(h)}^{i} \mathbf{d}_{i}, \forall(h) \in I_{m} .
$$

We have to note another very important point: all principal components represent a basis of ${ }_{\mathrm{d}} V_{(0)}^{m}$. In other words, all principal components represent basic risky assets. We currently denote such a basis by ${ }_{m} B_{\mathbf{w}_{\mathbf{d}}}$. Every risky asset belonging to ${ }_{\mathrm{d}} V_{(0)}^{m}$ can therefore be expressed as a linear combination of the basic risky assets belonging to ${ }_{m} B_{\mathbf{w}_{\mathbf{d}}}$. In particular, we then write

$$
\mathbf{d}_{i}=v_{i}^{(h)} \mathbf{w}_{(h)}, \forall i \in I_{m} .
$$


Having said that, we consider the following eigenequation

$$
\left({ }_{\mathbf{d}} g_{k h}-\lambda_{(k)} \delta_{k h}\right) v_{(k)}^{k}=0 .
$$

From (49) it follows

$$
{ }_{\mathbf{d}} g_{k h} v_{(k)}^{k}=\lambda_{(k)} \delta_{k h} v_{(k)}^{k} .
$$

If we use the contravariant components of $\mathbf{v}_{h}$ in both sides of (50) then we obtain

$$
v_{(k)}^{k} v_{(h) \mathbf{d}}^{h} g_{k h}=\lambda_{(k)} v_{(k)}^{k} v_{(h)}^{h} \delta_{k h} .
$$

On the other hand, it is also possible to write

$$
{ }_{\mathbf{d}} g_{k h}=v_{k}^{(j)} v_{h}^{(i)}\left\langle\mathbf{w}_{(j)}, \mathbf{w}_{(i)}\right\rangle_{\alpha}
$$

after considering (48). We observe that it turns out to be

$$
v_{(k)}^{k} v_{(h)}^{h} \delta_{k h}=\delta_{(k)(h)},
$$

so it is possible to obtain

$$
\left\langle\mathbf{w}_{(k)}, \mathbf{w}_{(h)}\right\rangle_{\alpha}=\lambda_{(k)} \delta_{(k)(h)} .
$$

We have obtained (54) after putting (52) in (51). If $(k)$ and $(h)$ vary in $I_{m}$ then we note that (54) identifies an $\alpha$-metric tensor with respect to a basis of ${ }_{\mathbf{d}} V_{(0)}^{m}$ whose elements are the principal components concerning $D_{\{m\}}$. We therefore write

$$
{ }_{\mathbf{w}} g_{(k)(h)}=\left\langle\mathbf{w}_{(k)}, \mathbf{w}_{(h)}\right\rangle_{\alpha}=\lambda_{(k)} \delta_{(k)(h)} .
$$

The generic covariant component of a diagonal tensor is expressed by (55). We have then shown that ${ }_{m} B_{\mathbf{w}_{\mathbf{d}}}$ is an $\alpha$-orthogonal basis of ${ }_{\mathbf{d}} V_{(0)}^{m}$. Its elements are pairwise $\alpha$-orthogonal. Also, every eigenvalue corresponding to the normalized eigenvector whose components are contained in (47) coincides with the $\alpha$-norm of a principal component belonging to ${ }_{m} B_{\mathbf{w}_{\mathbf{d}}}([23])$. Since it is possible to write

$$
\left[\begin{array}{cccc}
{ }_{\mathrm{w}} g_{(1)(1)} & { }_{\mathrm{w}} g_{(1)(2)} & \cdots & { }_{\mathrm{w}} g_{(1)(m)} \\
{ }_{\mathrm{w}} g_{(2)(1)} & { }_{\mathrm{w}} g_{(2)(2)} & \cdots & { }_{\mathrm{w}} g_{(2)(m)} \\
\vdots & \vdots & \ddots & \vdots \\
\mathrm{w} g_{(m)(1)} & { }_{\mathbf{w}} g_{(m)(2)} & \cdots & { }_{\mathrm{w}} g_{(m)(m)}
\end{array}\right],
$$

we denote by ${ }_{\mathbf{w}} a^{(k)(h)}$ the cofactor of ${ }_{\mathbf{w}} a_{(k)(h)}$, where both ${ }_{\mathbf{w}} a^{(k)(h)}$ and ${ }_{\mathbf{w}} a_{(k)(h)}$ are contained in (56). Also, we denote by ${ }_{\mathrm{w}} g$ the determinant of (56). The 
generic contravariant component of the $\alpha$-metric tensor under consideration is therefore given by

$$
{ }_{\mathbf{w}} g^{(k)(h)}=\frac{{ }_{\mathbf{w}} a^{(k)(h)}}{{ }_{\mathbf{w}} g} .
$$

If we write

$$
\frac{1}{\lambda_{(k)}}=\lambda^{(k)},
$$

then it is possible to obtain

$$
{ }_{\mathbf{w}} g^{(k)(h)}=\lambda^{(k)} \delta^{(k)(h)} .
$$

By putting together (55) and (58) we are lastly able to write

$$
{ }_{\mathbf{w}} g_{(k)(h) \mathbf{w}} g^{(k)(h)}=\lambda_{(k)} \delta_{(k)(h)} \lambda^{(k)} \delta^{(k)(h)}=\lambda_{(k)} \lambda^{(j)} \delta_{(k)}^{(j)} .
$$

We have obtained a mixed and $\alpha$-metric tensor whose generic component can be denoted by ${ }_{\mathrm{w}} g_{(k)}^{(j)}$. It follows that it turns out to be ${ }_{\mathrm{w}} g_{(k)}^{(k)}=m$ because we consider a product of matrices by means of which we obtain an $m \times m$ identity matrix of which we compute its trace.

\section{The projection of a linear manifold onto another one obtained when we choose a particular basis of it}

We have said that if we project $\mathbf{d}^{\prime} V_{(0)}^{m}$ onto ${ }_{\mathbf{d}} V_{(0)}^{m}$ then we obtain an $m$-dimensional linear manifold embedded in $E^{n}$ denoted by ${ }_{\hat{\mathbf{d}}} V_{(0)}^{m}$. We observe that ${ }_{m} B_{\mathbf{w}_{\mathbf{d}}}$ is a basis of it. This means that it is possible to write

$$
\hat{\mathbf{d}}_{j}=d_{j}^{(i)} \mathbf{w}_{(i)}, \forall j \in I_{m} .
$$

We remind (41). The following condition

$$
\left\langle\mathbf{d}_{j}^{\prime}, \mathbf{w}_{(k)}\right\rangle_{\alpha}-d_{j}^{(h)}{ }_{\mathbf{w}} g_{(h)(k)}=\left\langle\mathbf{d}_{j}^{\prime}, \mathbf{w}_{(k)}\right\rangle_{\alpha}-d_{j(k)}=0
$$

therefore allows us of computing the covariant components of $\hat{\mathbf{d}}_{j} \in \hat{\mathbf{d}}_{(0)}^{m}$ with respect to ${ }_{m} B_{\mathbf{w}_{\mathbf{d}}}$. Given (61), it turns out to be

$$
d_{j(k)}=\left\langle\mathbf{d}_{j}^{\prime}, \mathbf{w}_{(k)}\right\rangle_{\alpha} .
$$

We take (58) into account, so we write

$$
d_{j(k) \mathbf{w}} g^{(h)(k)}=d_{j(k)} \lambda^{(h)} \delta^{(h)(k)}=d_{j(h)} \lambda^{(h)} .
$$


We observe that the $h$ index in the third side of (63) is a free index unlike the $k$ index in the second side of it. The contravariant components of $\hat{\mathbf{d}}_{j} \in \hat{\mathbf{d}}_{(0)}^{m}$ with respect to ${ }_{m} B_{\mathbf{w}_{\mathbf{d}}}$ are then expressed by

$$
d_{j(h)} \lambda^{(h)}=\frac{\left\langle\mathbf{d}_{j}^{\prime}, \mathbf{w}_{(h)}\right\rangle_{\alpha}}{\lambda_{(h)}}=d_{j}^{(h)} .
$$

We lastly observe that the covariant components of $\hat{\mathbf{d}}_{j}$ and $\mathbf{d}_{j}^{\prime}$ with respect to ${ }_{m} B_{\mathbf{w}_{\mathbf{d}}}$ are obtained in the same way. This means that it is possible to write

$$
d_{j(k)}=\left\langle\hat{\mathbf{d}}_{j}, \mathbf{w}_{(k)}\right\rangle \alpha
$$

\section{A proportionality existing between risky assets: a particular case}

We have said that every risky asset subjected to a change of origin coincides with an $n$-dimensional vector of $E^{n}$ representing a marginal distribution of probability ([6]). Such a vector is an ordered set of real numbers. If we consider $m$ risky assets then we deal with $m$ ordered sets of real numbers because every risky asset is an ordered set of real numbers. We say that two ordered sets of two non-zero real numbers denoted by $\left\{d_{A}^{1}, d_{A}^{2}\right\}$ and $\left\{d_{B}^{1}, d_{B}^{2}\right\}$ are proportional if it is possible to write

$$
d_{A}^{1}: d_{B}^{1}=d_{A}^{2}: d_{B}^{2}
$$

This means that there exists a constant of proportionality denoted by $h$ such that we have

$$
\frac{d_{A}^{1}}{d_{B}^{1}}=\frac{d_{A}^{2}}{d_{B}^{2}}=h
$$

In general, given two ordered sets of $n$ real numbers denoted by $\left\{d_{A}^{1}, d_{A}^{2}, \ldots, d_{A}^{n}\right\}$ and $\left\{d_{B}^{1}, d_{B}^{2}, \ldots, d_{B}^{n}\right\}$, we say that they are proportional if it is possible to write

$$
\left(\begin{array}{c}
d_{A}^{1}=h d_{B}^{1} \\
d_{A}^{2}=h d_{B}^{2} \\
\vdots \\
d_{A}^{n}=h d_{B}^{n}
\end{array}\right)
$$


It follows that we are also able to write

$$
\left(\begin{array}{c}
d_{A}^{1}-d_{B}^{1}=(h-1) d_{B}^{1} \\
d_{A}^{2}-d_{B}^{2}=(h-1) d_{B}^{2} \\
\vdots \\
d_{A}^{n}-d_{B}^{n}=(h-1) d_{B}^{n}
\end{array}\right)
$$

Given the direct difference between $\left\{d_{A}^{1}, d_{A}^{2}, \ldots, d_{A}^{n}\right\}$ and a homothetic transformation of $\left\{d_{B}^{1}, d_{B}^{2}, \ldots, d_{B}^{n}\right\}$, if we say that such a difference is proportional to a third set of $n$ real numbers denoted by $\left\{d_{C}^{1}, d_{C}^{2}, \ldots, d_{C}^{n}\right\}$ then it is possible to write

$$
\left(\begin{array}{c}
d_{A}^{1}-x d_{B}^{1}=y d_{C}^{1} \\
d_{A}^{2}-x d_{B}^{2}=y d_{C}^{2} \\
\vdots \\
d_{A}^{n}-x d_{B}^{n}=y d_{C}^{n}
\end{array}\right)
$$

We note that $y$ is an average constant of proportionality. We suppose that all equalities expressed by (70) do not hold. We consequently establish a probabilistic and economic criterion by means of which it is possible to construct an ordered set of $n$ real numbers whose elements are given by $\left\{d_{C^{\prime}}^{1}, d_{C^{\prime}}^{2}, \ldots, d_{C^{\prime}}^{n}\right\}$. We realize that $\left\{d_{C^{\prime}}^{1}, d_{C^{\prime}}^{2}, \ldots, d_{C^{\prime}}^{n}\right\}$ must have got pre-established characteristics with respect to $\left\{d_{C}^{1}, d_{C}^{2}, \ldots, d_{C}^{n}\right\}$. We observe that the following equalities

$$
\left(\begin{array}{c}
d_{A}^{1}-x d_{B}^{1}=y d_{C^{\prime}}^{1} \\
d_{A}^{2}-x d_{B}^{2}=y d_{C^{\prime}}^{2} \\
\vdots \\
d_{A}^{n}-x d_{B}^{n}=y d_{C^{\prime}}^{n}
\end{array}\right)
$$

must then be satisfied. We lastly observe that what we will say in the next section makes more functional what we have said in this one.

\section{The condition of invariance of the covariance of two risky assets: a particular case}

We have considered the $\alpha$-orthogonal projection of ${ }_{\mathbf{d}^{\prime}} V_{(0)}^{m}$ onto ${ }_{\mathbf{d}} V_{(0)}^{m}$. We have denoted it by ${ }_{\hat{\mathbf{d}}} V_{(0)}^{m}$. All risky assets belonging to $\hat{\mathrm{d}}_{(0)}^{m}$ are then nothing but 
units of measurement with respect to which an investor is able to measure and characterize all risky assets belonging to ${ }_{\mathrm{d}} V_{(0)}^{m}$. To fix ideas, we suppose that it turns out to be $m=2$. We therefore write

$$
\mathbf{d}_{1}^{*}=\mathbf{d}_{1}^{\prime}-\hat{\mathbf{d}}_{1}
$$

and

$$
\mathbf{d}_{2}^{*}=\mathbf{d}_{2}^{\prime}-\hat{\mathbf{d}}_{2} .
$$

It follows that we have to consider two systems of two linear equations in order to construct $\hat{\mathrm{d}}_{(0)}^{m}$. We have

$$
\left\{\begin{array}{l}
\left\langle\mathbf{d}_{1}^{*}, \mathbf{d}_{1}\right\rangle_{\alpha}=0 \\
\left\langle\mathbf{d}_{1}^{*}, \mathbf{d}_{2}\right\rangle_{\alpha}=0
\end{array}\right.
$$

as well as

$$
\left\{\begin{array}{l}
\left\langle\mathbf{d}_{2}^{*}, \mathbf{d}_{1}\right\rangle_{\alpha}=0 \\
\left\langle\mathbf{d}_{2}^{*}, \mathbf{d}_{2}\right\rangle_{\alpha}=0
\end{array} .\right.
$$

If we consider (72) and (73) then we are also able to write

$$
\left\{\begin{array}{l}
\left\langle\mathbf{d}_{1}^{\prime}, \mathbf{d}_{1}\right\rangle_{\alpha}=\left\langle\hat{\mathbf{d}}_{1}, \mathbf{d}_{1}\right\rangle_{\alpha} \\
\left\langle\mathbf{d}_{1}^{\prime}, \mathbf{d}_{2}\right\rangle_{\alpha}=\left\langle\hat{\mathbf{d}}_{1}, \mathbf{d}_{2}\right\rangle_{\alpha}
\end{array}\right.
$$

as well as

$$
\left\{\begin{array}{l}
\left\langle\mathbf{d}_{2}^{\prime}, \mathbf{d}_{1}\right\rangle_{\alpha}=\left\langle\hat{\mathbf{d}}_{2}, \mathbf{d}_{1}\right\rangle_{\alpha} \\
\left\langle\mathbf{d}_{2}^{\prime}, \mathbf{d}_{2}\right\rangle_{\alpha}=\left\langle\hat{\mathbf{d}}_{2}, \mathbf{d}_{2}\right\rangle_{\alpha}
\end{array} .\right.
$$

Having said that, given two basic risky assets of ${ }_{\mathbf{d}} V_{(0)}^{m}$ denoted by $\mathbf{d}_{1}$ and $\mathbf{d}_{2}$, it is possible to consider

and

$$
\mathbf{d}_{1}-x \mathbf{d}_{2}=y \hat{\mathbf{d}}_{1}
$$

where $\hat{\mathbf{d}}_{1}$ and $\hat{\mathbf{d}}_{2}$ are two basic risky assets of $\hat{\mathbf{d}}_{(0)}^{m}$. We note that $y$ and $y^{\prime}$ are average constants of proportionality because they are referred to probability 
distributions, while $x$ and $x^{\prime}$ are coefficients of adjustment. They adjust the difference of $\mathbf{d}_{1}$ and $\mathbf{d}_{2}$ to distributions that should exist with respect to a probabilistic and economic hypothesis identifying the invariance of the covariance of two risky assets. We deal with a Bayesian adjustment because $\mathbf{d}_{1}$ and $\mathbf{d}_{2}$ are two prior distributions, while $\hat{\mathbf{d}}_{1}$ and $\hat{\mathbf{d}}_{2}$ are two posterior distributions characterizing a specific hypothesis ([1]). We then observe that a distance between two prior distributions is proportional to a posterior distribution characterizing the right-hand side of (78). A distance between two prior distributions is similarly proportional to a posterior distribution appearing in the right-hand side of (79) ([13]). We note that $\hat{\mathbf{d}}_{1}$ in (78) and $\hat{\mathbf{d}}_{2}$ in (79) are obtained by means of linear combinations of $\mathbf{d}_{1}$ and $\mathbf{d}_{2}$. It is then possible to refer to (40). The condition of invariance of the covariance of two risky assets expressed by (76) and (77) is equal to the condition according to which $\mathbf{d}_{1}^{*}=\mathbf{d}_{1}^{\prime}-\hat{\mathbf{d}}_{1}$ and $\mathbf{d}_{2}^{*}=\mathbf{d}_{2}^{\prime}-\hat{\mathbf{d}}_{2}$ are orthogonal to the plane established by $\mathbf{d}_{1}$ and $\mathbf{d}_{2}$. We speak about plane because we deal with two risky assets of which we compute their covariance. It is indeed possible to show that two risky assets coinciding with two random quantities identify a geometric shape in 2 dimensions. It is a parallelogram with two pairs of parallel sides, where every risky asset coinciding with a random quantity is a side of it. Such a parallelogram recognizes a bivariate risky asset coinciding with a bivariate random quantity. We observe that $\hat{\mathbf{d}}_{1}$ coincides with the orthogonal projection of $\hat{\mathbf{d}}_{1}$ onto $\mathbf{d}_{1}^{\prime}$ given by

$$
\operatorname{proj}_{\mathbf{d}^{\prime}{ }_{1}}\left(\hat{\mathbf{d}}_{1}\right)=\frac{\mathbf{d}_{1}^{\prime} \cdot \hat{\mathbf{d}}_{1}}{\left\|\mathbf{d}_{1}^{\prime}\right\|^{2}} \mathbf{d}_{1}^{\prime}
$$

as well as $\hat{\mathbf{d}}_{2}$ coincides with the orthogonal projection of $\hat{\mathbf{d}}_{2}$ onto $\mathbf{d}_{2}^{\prime}$ expressed by

$$
\operatorname{proj}_{\mathbf{d}^{\prime} 2}\left(\hat{\mathbf{d}}_{2}\right)=\frac{\mathbf{d}_{2}^{\prime} \cdot \hat{\mathbf{d}}_{2}}{\left\|\mathbf{d}_{2}^{\prime}\right\|^{2}} \mathbf{d}_{2}^{\prime} .
$$

On the other hand, $\mathbf{d}_{1}^{\prime}$ coincides with the orthogonal projection of $\mathbf{d}_{1}^{\prime}$ onto $\hat{\mathbf{d}}_{1}$ as well as $\mathbf{d}_{2}^{\prime}$ coincides with the orthogonal projection of $\mathbf{d}_{2}^{\prime}$ onto $\hat{\mathbf{d}}_{2}$.

\section{Mean-variance utility}

Given a portfolio having two different types of asset, one of them is not a risky asset but it is a risk-free asset ([19]). It always pays a given amount of money regardless of what happens. We then say that its return is a positive constant, while its standard deviation is equal to 0 because there is not riskiness. The other asset is a set of $m$ risky assets. They give origin to a multivariate risky 
asset of order $m$. The mean-variance model assumes that the utility of a distribution of probability can be expressed as a function of the mean and variance of it ([17]). It is appropriate to make the natural assumption that a higher expected return on wealth is good when all other things do not change. A higher variance is conversely bad. This evidently means that the natural assumption of aversion to risk holds. We then suppose that there exists an inverse linear relationship between these two different types of asset ([20]). It is possible to assume that an investor's preferences depend only on the mean and variance of the distribution of probability of his wealth. It is then possible to consider indifference curves illustrating an investor's preferences for return and risk. If he is a risk-averse investor then a higher expected return on wealth makes him better off as well as a higher standard deviation makes him worse off. Riskiness represented by the variance of the distribution of probability of his wealth is a bad, so the indifference curves characterizing his utility function must have a positive slope $([25])$. We are able to describe the distribution of probability of a multivariate risky asset of order $m$ by using a few parameters. We are therefore interested in summarizing the distribution of probability of a multivariate risky asset of order $m$. This is because the utility function characterizing the mean-variance model must be defined over those parameters concerning such a distribution. An investor's preferences can therefore be described by considering just a few summary statistics about probability distribution of a multivariate risky asset of order $m$. On the other hand, we have shown that it is possible to decompose it in a linear space provided with a Euclidean metric. Having said that, we write

$$
\mathbf{P}\left(X_{1} X_{2} \cdots X_{m}\right)=\mathbf{P}\left(X_{1}\right) \mathbf{P}\left(X_{2}\right) \cdots \mathbf{P}\left(X_{m}\right),
$$

where $\mathbf{P}$ denotes the expected return on wealth. It coincides with a coherent prevision of a random quantity, where we evidently consider both a multivariate random quantity of order $m$ and $m$ random quantities. We also write

$$
\sigma_{X_{1}+X_{2}+\ldots+X_{m}}^{2}=\sigma_{X_{1}}^{2}+\sigma_{X_{2}}^{2}+\ldots+\sigma_{X_{m}}^{2}
$$

in order to obtain the variance of the probability distribution of a multivariate risky asset of order $m$. We observe that it also turns out to be

$$
\sigma_{X_{1}+X_{2}+\ldots+X_{m}}^{2}={ }_{\mathrm{d}} g_{11}+{ }_{\mathrm{d}} g_{22}+\ldots+{ }_{\mathrm{d}} g_{m m}
$$

as well as

$$
\sigma_{X_{1}+X_{2}+\ldots+X_{m}}=\sqrt{\mathbf{d} g_{11}}+\sqrt{\mathbf{d} g_{22}}+\ldots+\sqrt{\mathbf{d} g_{m m}},
$$

where we evidently consider the sum of elements on the main diagonal of (25) as well as the sum of their square roots. At the optimal choice of mean return and 
standard deviation of return of the probability distribution of a multivariate risky asset of order $m$ we observe that the slope of the indifference curve must be equal to the slope of the budget line. Such a line measures the cost of obtaining a larger expected return in terms of the increased standard deviation of the return. Its vertical intercept coincides with the return of the risk-free asset under consideration.

\section{Some final remarks}

It is possible to show that the notion of direction of an appropriate vector belonging to a linear space over $\mathbb{R}$ has a probabilistic and economic meaning. Particular equations of proportionality are used in order to show this thing. It is consequently possible to compute the coefficients connected with these particular equations of proportionality. It is also possible to prove a theorem of $\alpha$-orthogonality that tells us that all basic risky assets belonging to $\hat{\mathbf{d}}_{(0)}^{m}$ coincide with the principal components. It is possible to show that the principal components have a probabilistic and economic meaning when they derive from particular equations of proportionality. It is possible to use a different approach to what we have shown in this paper. It is therefore possible to use Grassmann coordinates of linear manifolds. They coincide with the components of an antisymmetric tensor whose order is equal to the dimension of the linear manifold under consideration. It is possible that all possible values of a multivariate risky asset of order $m$ coincide with the components of an antisymmetric tensor of order $m$.

\section{Conclusions}

We have analyzed $m$ risky assets coinciding with $m$ marginal distributions of probability inside of a linear space. They generate a distribution of probability of a multivariate risky asset of order $m$. We have decomposed such a distribution inside of a linear space. We have shown that an $m$-dimensional linear manifold is generated by $m$ basic risky assets. Given $m$ basic risky assets, we have proved that all risky assets contained in an $m$-dimensional linear manifold are geometrically related. We have proved that two any risky assets of them are conversely $\alpha$-orthogonal, so their covariance is equal to 0 . We have reinterpreted principal component analysis by showing that the principal components are basic risky assets of an $m$-dimensional linear manifold. We have considered a Bayesian adjustment of differences between prior distributions to posterior 
distributions existing with respect to a probabilistic and economic hypothesis identifying the invariance of the covariance of two risky assets.

\section{References}

[1] E.W. Anderson, A.R. Cheng, Robust Bayesian portfolio choices, The Review of Financial Studies, 29, No 5 (2016), 1330-1375.

[2] G. Coletti, D. Petturiti, B. Vantaggi, When upper conditional probabilities are conditional possibility measures, Fuzzy Sets and Systems, 304 (2016), $45-64$.

[3] G. Coletti, D. Petturiti, B. Vantaggi, Bayesian inference: the role of coherence to deal with a prior belief function, Statistical Methods $\&$ Applications, 23, No 4 (2014), 519-545.

[4] B. de Finetti, The proper approach to probability, In: Exchangeability in Probability and Statistics, North-Holland Publishing Company, Amsterdam (1982), 1-6.

[5] B. de Finetti, Probabilism: A critical essay on the theory of probability and on the value of science, Erkenntnis, 31, No 2-3 (1989), 169-223.

[6] B. de Finetti, Probability: beware of falsifications!, In: Studies in Subjective Probability, R. E. Krieger Publishing Company, Huntington, New York (1980), 195-224.

[7] B. de Finetti, The role of "Dutch Books" and of "proper scoring rules", The British Journal of Psychology of Sciences, 32 (1981), 55-56.

[8] B. de Finetti, Probability: the different views and terminologies in a critical analysis, In: Logic, Methodology and Philosophy of Science VI, NorthHolland Publishing Company, Amsterdam (1982), 391-394.

[9] L.G. Epstein, J. Zhang, Subjective probabilities on subjectively unambiguous events, Econometrica, 69, No 2 (2001), 265-306.

[10] A. Gilio, G. Sanfilippo, Conditional random quantities and compounds of conditionals, Studia Logica, 102, No 4 (2014), 709-729.

[11] B. Grechuk, A. Molyboha, M. Zabarankin, Mean-deviation analysis in the theory of choice, Risk Analysis: An International Journal, 32, No 8 (2012), 1277-1292. 
[12] B. Grechuk, M. Zabarankin, Inverse portfolio problem with mean-deviation model, European Journal of Operational Research, 234, No 2 (2014), 481490.

[13] Y. Halevy, V. Feltkamp, A Bayesian approach to uncertainty aversion, Review of Economic Studies, 72, No 2 (2005), 449-466.

[14] H. Hotelling, Relations between two sets of variates, Biometrika, 28, No 3-4 (1936), 321-377.

[15] I.T. Jolliffe, J. Cadima, Principal component analysis: a review and recent developments, Philosophical Transactions of the Royal Society A: Mathematical, Physical and Engineering Sciences, 374, No 2065 (2016), 1-16.

[16] E.J. Johnson, J.W. Payne, Effort and accuracy in choice, Management Science, 31, No 4 (1985), 395-414.

[17] F. Maccheroni, M. Marinacci, A. Rustichini, M. Taboga, Portfolio selection with monotone mean-variance preferences, Mathematical Finance, 19, No 3 (2009), 487-521.

[18] M. Machina, D. Schmeidler, A more robust definition of subjective probability, Econometrica, 60, No 4 (1992), 745-780.

[19] H. M. Markowitz, Portfolio selection, The Journal of Finance, 7, No 1 (1952), 77-91.

[20] H. M. Markowitz, The optimization of a quadratic function subject to linear constraints, Naval Research Logistics Quarterly, 3, No 1-2 (1956), 111-133.

[21] G. Pasini, Principal component analysis for stock portfolio management, International Journal of Pure and Applied Mathematics, 115, No 1 (2017), $153-167$.

[22] G. Pompilj, On intrinsic independence, Bulletin of the International Statistical Institute, 35, No 2 (1957), 91-97.

[23] C.R. Rao, The use and interpretation of principal component analysis in applied research, Sankhya: the Indian Journal of Statistics, Series A, 26, No 4 (1964), 329-358. 
[24] R.T. Rockafellar, S. Uryasev, M. Zabarankin, Optimality conditions in portfolio analysis with general deviation measures, Mathematical Programming, 108, No 2-3 (2006), 515-540.

[25] M.E. Yaari, The dual theory of choice under risk, Econometrica, 55, No 1 (1987), 95-115. 
